\title{
CONVERGENCE OF TRUNCATED SINGULAR INTEGRALS WITH TWO WEIGHTS
}

\author{
BY \\ LILIANA DE ROS A AND CARLOS SEgOVIA (BUENOS AIRES)
}

1. Introduction. By $\mathbf{R}^{n}$ we denote the $n$-dimensional euclidean space and for $x$ and $y$ in $\mathbf{R}^{n}$ we set $(x \cdot y)=\sum_{i=1}^{n} x_{i} y_{i}$ and $|x|=(x \cdot x)^{1 / 2}$. If $E$ is a Lebesgue measurable set contained in $\mathbf{R}^{n},|E|$ stands for its Lebesgue measure. We shall say that an ordered pair of non-negative measurable functions $(v(x), u(x))$ defined on $\mathrm{R}^{n}$ belongs to the class $A(p, q), 1 \leq p \leq \infty$ and $1 \leq q \leq \infty$, if there exists a finite constant $C$ such that for every ball (or every cube) $B \subset \mathbf{R}^{n}$

$$
\left(|B|^{-1} \int_{B} u(x)^{-p^{\prime}} d x\right)^{1 / p^{\prime}}\left(|B|^{-1} \int_{B} v(x)^{q} d x\right)^{1 / q} \leq C .
$$

Here and in the sequel, $p^{\prime}$ denotes the conjugate exponent of $p$, i.e. $p^{\prime}=$ $p /(p-1)$.

Let $w(x)$ be a non-negative measurable function. We denote by $L^{p}(w)$, $1 \leq p \leq \infty$, the class of measurable functions $f$ such that

$$
\|f\|_{L^{p}(w)}=\left(\int_{\mathbf{R}^{n}}|f(x)|^{p} w(x) d x\right)^{1 / p}<\infty .
$$

If $w=1$, we simply write $L^{p}$. The element of surface area of the unit sphere $\Sigma=\left\{x:|x|=1, x \in \mathbf{R}^{n}\right\}$ will be denoted by $d \sigma(x)$. The space $L^{p}(\Sigma)$, $1 \leq p \leq \infty$, is the class of all measurable functions $f$ defined on $\Sigma$ such that

$$
\|f\|_{L^{p}(\Sigma)}=\left(\int_{\Sigma}|f(x)|^{p} d \sigma(x)\right)^{1 / p}<\infty .
$$

Let $k(x)=\Omega(x) /|x|^{n}$ be a function defined on $\mathbf{R}^{n} \backslash\{0\}$. We shall say that $k$ is an $L^{r}$-Dini singular integral kernel (see [2] and [4]) if $\Omega(x)$ satisfies the following assumptions:

(i) $\Omega(x)$ is a positively homogeneous function of degree zero, 
(ii) $\Omega \in L^{r}(\Sigma), 1 \leq r \leq \infty$, and if

$$
w_{r}(\delta)=\sup _{|\rho|<\delta}\left(\int_{\Sigma}|\Omega(\rho x)-\Omega(x)|^{r} d \sigma(x)\right)^{1 / r},
$$

where $\rho$ denotes a rotation and $|\rho|=\sup _{x \in \Sigma}|\rho x-x|$, then

$$
\int_{0}^{1} w_{r}(\delta) \delta^{-1} d \delta<\infty,
$$

(iii) $\int_{\Sigma} \Omega(x) d \sigma(x)=0$.

Let $k$ be an $L^{r}$-Dini singular integral kernel and $\eta>0$. The function $k_{\eta}(x)$ defined as

$$
\begin{array}{ll}
k_{\eta}(x)=k(x) & \text { if }|x| \geq \eta, \\
k_{\eta}(x)=0 & \text { otherwise, }
\end{array}
$$

will be called the truncated singular integral kernel of $k$. The truncated singular integral of a function $f$ is defined as

$$
K_{\eta}(f)(x)=\int_{\mathbf{R}^{n}} k_{\eta}(x-y) f(y) d y=\int_{|x-y|>\eta} k(x-y) f(y) d y,
$$

and the singular integral of $f$ is defined as the limit .

$$
K(f)(x)=\lim _{\eta \rightarrow 0} K_{\eta}(f)(x)=\lim _{\eta \rightarrow 0} \int_{|x-y|>\eta} k(x-y) f(y) d y
$$

whenever this limit exists.

The main result of this paper is the following theorem:

(1.2) TheOREM A. Let $k(x)$ be an $L^{r}$-Dini singular integral kernel and $(v, u) \in A\left(p, p(r / p)^{\prime}\right), 1 \leq p \leq r<\infty$. If $f$ and $K(f)$ belong to $L^{p}\left(u^{p}\right)$, then

$$
\left\|K_{\varepsilon}(f)\right\|_{L^{p}\left(v^{p}\right)} \leq C\left\{\|f\|_{L^{p}\left(u^{p}\right)}+\|K(f)\|_{L^{p}\left(u^{p}\right)}\right\}
$$

for every $\varepsilon>0$ with a constant $C$ not depending on $\varepsilon$ and $f$. Moreover,

$$
\lim _{\varepsilon \rightarrow 0}\left\|K_{\varepsilon}(f)-K(f)\right\|_{L^{p}\left(v^{p}\right)}=0 \text {. }
$$

For the case $v=u, 1=p<r$, Theorem A is known (see [3]), for $1<p<r$; the continuity of the maximal singular integral $K^{*}$ holds and implies Theorem A (see [1] and [3]). In the case of different weights, $v \neq u$, we do not even have in general the continuity of $K(f)$. Thus, the proof of Theorem A does not distinguish whether $p$ is equal to one or not. We shall use in the proof a generalization of a remarkable result of B. Muckenhoupt (see [5]) which we believe has an independent interest; we state it in the following theorem: 
(1.3) TheOREM B. Let $F(x)$ be a function defined on $\mathbf{R}^{n}$. For $k \in \mathbf{Z}$, set

$$
c_{k}=\left(\int_{2^{k}<|x| \leq 2^{k+1}}|F(x)|^{r} d x\right)^{1 / r} \text {. }
$$

Assume that

$$
A=\sum_{k \in \mathbf{Z}} c_{k} 2^{k n / r^{\prime}}<\infty
$$

Then, if $F_{\varepsilon}(x)=\varepsilon^{-n} F(x / \varepsilon), 1 \leq p<r \leq \infty$ or $1 \leq p=r<\infty,(v, u) \in$ $A\left(p, p(r / p)^{\prime}\right)$ and $f \in L^{p}\left(u^{p}\right)$, we have

$$
\left\|F_{\varepsilon} * f\right\|_{L^{p}\left(v^{p}\right)} \leq c A\|f\|_{L^{p}\left(u^{p}\right)}
$$

with a constant $c$ depending on $n, r, p$ and the constant $C$ of (1.1) only. Moreover, for $a=\int_{\mathbf{R}^{n}} F(x) d x$,

$$
\lim _{\varepsilon \rightarrow 0}\left\|F_{\varepsilon} * f-a f\right\|_{L p(v p)}=0 \text {. }
$$

2. Proof of the Muckenhoupt-type theorem on the approximation of the identity. The main property of the $L^{r}$-Dini singular integral kernels that will be used in the sequel is the following lemma due to D. S. Kurtz and R. L. Wheeden, see [4]:

(2.1) LеммA. Let $k(x)$ be an $L^{r}$-Dini singular integral kernel and $|y|<$ $R / 2$. Then

$$
\begin{aligned}
\left(\int_{R<|x|<2 R}|k(x-y)-k(x)|^{r} d x\right)^{1 / r} \\
\quad \leq c R^{-n / r^{\prime}}\left(|y| / R+\int_{|y| / 2 R<\delta<|y| / R} w_{r}(\delta) \delta^{-1} d \delta\right),
\end{aligned}
$$

where $c$ does not depend on $R>0$.

Proof of Theorem B, (1.3). (i) Let $\chi_{k}$ be the characteristic function of the annulus $2^{k}<|x| \leq 2^{k+1}, k \in \mathbf{Z}$. Let $\left\{Q_{j}\right\}$ be a partition of $\mathbf{R}^{n}$ into cubes with sides of length $2^{k+1} \varepsilon$. Then we have

$$
\begin{aligned}
& \left\|\left(F \chi_{k}\right)_{\varepsilon} * f\right\|_{L^{p}\left(v^{p}\right)}^{p} \\
& \quad \leq \varepsilon^{-n p} \sum_{j} \int_{Q_{j}}\left(\int\left|\left(F \chi_{k}\right)((z-x) / \varepsilon)\right||f(x)| d x\right)^{p} v(z)^{p} d z .
\end{aligned}
$$

By Minkowski's integral inequality, (2.2) is bounded by

$$
\varepsilon^{-n p} \sum_{j}\left[\int_{3 Q_{j}}|f(x)|\left(\int_{Q_{j}}\left|\left(F \chi_{k}\right)((z-x) / \varepsilon)\right|^{p} v(z)^{p} d z\right)^{1 / p} d x\right]^{p} .
$$


For each $j$, by Hölder's inequality, we have

$$
\begin{aligned}
& \left(\int_{Q_{j}}\left|\left(F \chi_{k}\right)((z-x) / \varepsilon)\right|^{p} v(z)^{p} d z\right)^{1 / p} \\
& \quad \leq \varepsilon^{n / r}\left(\int_{2^{k}<|z| \leq 2^{k+1}}|F(z)|^{r} d z\right)^{1 / r}\left(\int_{Q_{j}} v(z)^{p(r / p)^{\prime}} d z\right)^{1 / p(r / p)^{\prime}} \\
& \quad=\varepsilon^{n / r} c_{k}\left(\int_{Q_{j}} v(z)^{p(r / p)^{\prime}} d z\right)^{1 / p(r / p)^{\prime}} .
\end{aligned}
$$

Thus, (2.2) is bounded by

$$
\varepsilon^{-n p / r^{\prime}} c_{k}^{p} \sum_{j}\left(\int_{Q_{j}} v(z)^{p(r / p)^{\prime}} d z\right)^{1 /(r / p)^{\prime}}\left(\int_{3 Q_{j}}|f(x)| d x\right)^{p} .
$$

Since, by Hölder's inequality,

$$
\left(\int_{3 Q_{j}}|f(x)| d x\right)^{p} \leq\left(\int_{3 Q_{j}}|f(x)|^{p} u(x)^{p} d x\right)\left(\int_{3 Q_{j}} u(x)^{-p^{\prime}} d x\right)^{p / p^{\prime}}
$$

and taking into account that $(v, u) \in A\left(p, p(r / p)^{\prime}\right)$, it follows that (2.2) is bounded by a constant times

$$
c_{k}^{p} 2^{k n p / r^{\prime}}\|f\|_{L^{p}\left(u^{p}\right)}^{p} .
$$

Then, by Minkowski's inequality, we obtain

$$
\left\|F_{\varepsilon} * f\right\|_{L^{p}\left(v^{p}\right)} \leq \sum_{k \in \mathbf{Z}}\left\|\left(F \chi_{k}\right)_{\epsilon} * f\right\|_{L^{p}\left(u^{p}\right)} \leq c\left(\sum_{k \in \mathbf{Z}} c_{k} 2^{k n / r^{\prime}}\right)\|f\|_{L^{p}\left(u^{p}\right)} .
$$

(ii) Assume first that $f$ is a bounded function with bounded support and $N$ is a number large enough so that $|x| \leq N$ contains the support of $f$, and

$$
\int_{|x| \leq N} v(x)^{p} d x>0, \quad \int_{|x| \leq N} u(x)^{-p^{\prime}} d x>0 .
$$

We observe that it is always possible to find $N$ unless $v=0$ a.e. or $u=\infty$ a.e., the cases that are trivial. We have

$$
\begin{aligned}
& \left\|F_{\varepsilon} * f-a f\right\|_{L^{p}(v p)}^{p} \\
& \quad \leq\left(\int_{|x| \leq 3 N}+\int_{|x|>3 N}\right)\left|F_{\varepsilon} * f-a f\right|^{p} v^{p} d x=I_{1}^{p}(\varepsilon)+I_{2}^{p}(\varepsilon) .
\end{aligned}
$$

Given $\eta>0$, let $M$ satisfy

$$
\int_{|z|>M}|F(z)| d z<\eta
$$


Such an $M$ exists since our assumption on $\left\{c_{k}\right\}$ implies that $F$ is integrable on $\mathbf{R}^{n}$. From the fact that $v^{p}$ is locally integrable and that $f$ is a bounded function with bounded support it follows easily that

$$
\left(\int|f(x-\varepsilon z)-f(x)|^{p} v(x)^{p} d x\right)^{1 / p}<\eta
$$

if $\varepsilon$ is small enough. Then

$$
\begin{aligned}
I_{1}(\varepsilon) \leq & \left(\int_{|x| \leq 3 N}\left(\int_{|z|>M}|F(z)||f(x-\varepsilon z)-f(x)| d z\right)^{p} v(x)^{p} d x\right)^{1 / p} \\
& +\left(\int_{|x| \leq 3 N}\left(\int_{|z| \leq M}|F(z)||f(x-\varepsilon z)-f(x)| d z\right)^{p} v(x)^{p} d x\right)^{1 / p} .
\end{aligned}
$$

Since $f$ is bounded, applying Minkowski's integral inequality we get

$$
\begin{aligned}
I_{1}(\varepsilon) \leq & 2\|f\|_{\infty} \int_{|z|>M}|F(z)| d z\left(\int_{|x| \leq 3 N} v(x)^{p} d x\right)^{1 / p} \\
& +\int_{|z| \leq M}|F(z)|\left(\int|f(x-\varepsilon z)-f(x)|^{p} v(x)^{p} d x\right)^{1 / p} d z \\
& \leq\left\{2\|f\|_{\infty}\left(\int_{|x| \leq 3 N} v(x)^{p} d x\right)^{1 / p}+\|F\|_{L^{1}}\right\} \eta
\end{aligned}
$$

showing that $I_{1}(\varepsilon)$ tends to zero with $\varepsilon$. Let us estimate $I_{2}(\varepsilon)$. By Minkowski's integral inequality, we have

$$
\begin{aligned}
& \left(\int_{|x|>3 N}\left|\left(F \chi_{k}\right)_{\varepsilon} * f\right|^{p} v^{p} d x\right)^{1 / p} \\
& \leq \varepsilon^{-n} \int|f(z)|\left(\int_{|x|>3 N}\left|\left(F \chi_{k}\right)((x-z) / \varepsilon)\right|^{p} v(x)^{p} d x\right)^{1 / p} d z .
\end{aligned}
$$

If $z$ belongs to the support of $f$ and $|x|>3 N$ it follows that $|z| \leq N$ and $|x-z|>2 N$. Then, the right hand side of (2.3) is equal to zero for $\varepsilon 2^{k+1} \leq 2 N$, that is to say, for $k \leq \log _{2}(N / \varepsilon)$. For $k>\log _{2}(N / \varepsilon)$, by Hölder's inequality, we get

$$
\left(\int_{|x|>3 N}\left|\left(F \chi_{k}\right)((x-z) / \varepsilon)\right|^{p} v(x)^{p} d x\right)^{1 / p}
$$

$$
\leq \varepsilon^{n / r}\left(\int_{2^{k}<|z| \leq 2^{k+1}}|F(z)|^{r} d z\right)^{1 / r}\left(\int_{|x-z| \leq 2^{k+1} \epsilon} v(x)^{p(r / p)^{\prime}} d x\right)^{1 / p(r / p)^{\prime}} .
$$

Multiplying and dividing by $\left(\int_{|x|<N} u(x)^{-p^{\prime}} d x\right)^{1 / p^{\prime}}$ and taking into account 
that $|x| \leq N$ implies $|x-z|<2^{k+1} \varepsilon$ whenever $|z|<N$, we find that (2.4) is bounded by

$$
c \varepsilon^{n} c_{k} 2^{k n / r^{\prime}}\left(\int_{|x|<N} u(x)^{-p^{\prime}} d x\right)^{-1 / p^{\prime}} .
$$

Thus, for (2.3) we have the bound

$$
c\|f\|_{L^{1}}\left(\int_{|x|<N} u(x)^{-p^{\prime}} d x\right)^{-1 / p^{\prime}} c_{k} 2^{k n / r^{\prime}} .
$$

This shows that $I_{2}(\varepsilon)$ is smaller than a constant times $\sum_{k>\log _{2}(N / \varepsilon)} c_{k} 2^{k n / r^{\prime}}$, which goes to zero with $\varepsilon$. We have shown that (ii) holds for functions $f$ bounded and with bounded support.

It is easy to see that the functions $g$ in $L^{p}\left(u^{p}\right)$ bounded and with bounded support are dense in $L^{p}\left(u^{p}\right)$. On the other hand, we observe that by the Lebesgue differentiation theorem, the condition $(v, u) \in A\left(p, p(r / p)^{\prime}\right)$ implies that $v(x) \leq c u(x)$ a.e. Thus,

$$
\begin{aligned}
& \left\|F_{\varepsilon} * f-a f\right\|_{L p(v p)} \\
\leq & \left\|F_{\varepsilon} *(f-g)\right\|_{L p(v p)}+\left\|F_{\varepsilon} * g-a g\right\|_{L p(v p)}+|a|\|f-g\|_{L p(v p)} .
\end{aligned}
$$

Then, by parts (i) and (ii) already proved for bounded functions with bounded support, we see that (2.5) is bounded by

$$
c\|f-g\|_{L p\left(u^{p}\right)}+\eta
$$

if $\varepsilon$ is close to zero. The density of the functions $g$ in $L^{p}\left(u^{p}\right)$ completes the proof of (ii).

(2.6) Corollary. Let $H(x)$ be a positively homogeneous function of degree zero belonging to $L^{r}(\Sigma), 1 \leq r \leq \infty$, and $\varphi$ a function with least decreasing radial majorant function $\psi(|\varphi(x)| \leq \psi(|x|))$ integrable on $\mathbf{R}^{n}$. If $(v, u)$ belongs to $A\left(p, p(r / p)^{\prime}\right)$ and $f \in L^{p}\left(u^{p}\right), 1 \leq p<r \leq \infty$ or $1 \leq p=r<\infty$, then

$$
\left\|(H \varphi)_{\epsilon} * f\right\|_{L^{p}\left(u^{p}\right)} \leq c\|H\|_{L^{r}(\Sigma)} \int_{0}^{\infty} \psi(t) t^{n-1} d t \cdot\|f\|_{L^{p}\left(u^{p}\right)},
$$

and moreover if $a=\int_{\mathbb{R}^{*}} H(x) \varphi(x) d x$, then

$$
\lim _{\varepsilon \rightarrow 0}\left\|(H \varphi)_{\varepsilon} * f-a f\right\|_{L p(v p)}=0 \text {. }
$$

P roof. Let $F=H \varphi$. For this $F$ we estimate the sequence $c_{k}$ of Theorem $B,(1.3)$. We have

$$
c_{k}=\left(\int_{2^{k}<|x| \leq 2^{k+1}}|(H \varphi)(x)|^{r} d x\right)^{1 / r} \leq c_{n, r} \psi\left(2^{k}\right)\|H\|_{L^{r}(\Sigma)} \cdot 2^{k n / r} .
$$


Thus,

$$
\begin{aligned}
\sum_{k \in \mathbf{Z}} c_{k} 2^{k n / r^{\prime}} & \leq c_{n, r}\|H\|_{L^{r}(\Sigma)} \sum_{k \in \mathbf{Z}} \psi\left(2^{k}\right) 2^{k n} \\
& \leq c_{n, r}^{\prime}\|H\|_{L^{r}(\Sigma)} \int_{0}^{\infty} \psi(t) t^{n-1} d t .
\end{aligned}
$$

(2.7) Corollary. Let $k(x)$ be an $L^{r}$-Dini singular integral kernel and $\varphi(x) \in C^{1}$ a function supported in the unit ball with $\int \varphi(x) d x=1$. Define

$$
\delta(x)=K(\varphi)(x)-k_{1}(x),
$$

where $k_{1}(x)=k(x)$ if $|x| \geq 1$ and $k_{1}(x)=0$ otherwise. Then the kernel $\delta_{\varepsilon}(x)=\varepsilon^{-n} \delta(x / \varepsilon)$ satisfies

$$
\begin{gathered}
\left\|\delta_{\varepsilon} * f\right\|_{L^{p}\left(v^{p}\right)} \leq c\|f\|_{L^{p}\left(u^{p}\right),} \\
\lim _{\varepsilon \rightarrow 0}\left\|\delta_{\varepsilon} * f\right\|_{L^{p}\left(v^{p}\right)}=0,
\end{gathered}
$$

whenever $(v, u)$ belongs to $A\left(p, p(r / p)^{\prime}\right), 1 \leq p<r \leq \infty$ or $1 \leq p=r<\infty$.

P r o of. Let $\delta^{(1)}(x)=\delta(x)$ for $|x| \leq 4$ and $\delta^{(1)}(x)=0$ if $|x| \geq 4$. Since $\varphi \in C^{1}$ it follows that $\left|\delta^{(1)}(x)\right| \leq c(1+|\Omega(x)|)$ for $|x| \leq 4$. Then, by Corollary (2.6), we find that $\delta^{(1)}$ satisfies (i). Let $\delta^{(2)}(x)=\delta(x)-\delta^{(1)}(x)$. Then we have

$$
\left|\delta^{(2)}(x)\right| \leq\|\varphi\|_{\infty} \int_{|y| \leq 1}|k(x-y)-k(x)| d y .
$$

Let us estimate the sequence $c_{k}$ of Theorem B, (1.3), for $F(x)=\delta^{(2)}(x)$. We observe that $c_{k}=0$ for $k<2$. In the case $k \geq 2$, we have

$$
\begin{aligned}
c_{k} & \leq c\left(\int_{2^{k}<|x| \leq 2^{k+1}}\left(\int_{|y| \leq 1}|k(x-y)-k(x)| d y\right)^{r} d x\right)^{1 / r} \\
& \leq c \int_{|y| \leq 1}\left(\int_{2^{k}<|x| \leq 2^{k+1}}|k(x-y)-k(x)|^{r} d x\right)^{1 / r} d y .
\end{aligned}
$$

Thus, by Lemma (2.1), we get

$$
c_{k} \leq c \cdot 2^{-k n / r^{\prime}}\left(2^{-k}+w_{r}\left(2^{-k}\right)\right)
$$

Therefore,

$$
\sum_{k \in Z} c_{k} 2^{k n / r^{\prime}} \leq c \sum_{k \geq 2}\left(2^{-k}+w_{r}\left(2^{-k}\right)\right)=c\left(1+\int_{0}^{1} w_{r}(t) d t / t\right)<\infty
$$

Thus, we can apply Theorem $B,(1.3)$, to $F=\delta^{(2)}$ and obtain part (i) of Corollary (2.7) for $\delta^{(2)}$. This, together with the result already obtained for $\delta^{(1)}$, proves (i) for $\delta=\delta^{(1)}+\delta^{(2)}$. As for part (ii), it is enough to prove that 
$\int \delta(x) d x=0$. Let $N>0$. Then, since $\int_{\Sigma} k(x) d \sigma(x)=0$ and recalling that $\varphi \in C^{1}$, we have

$$
\begin{aligned}
\int_{|x|<N} \delta(x) d x & =\int_{|x|<N} K(\varphi)(x) d x \\
& =\int_{|x|<N} d x \int_{|y|<N+1} k(y)[\varphi(x-y)-\varphi(x)] d y .
\end{aligned}
$$

Changing the order of integration, we get

$$
\begin{aligned}
\int_{|x|<N} \delta(x) d x & =\int_{|y|<N+1} k(y)\left(\int_{|x|<N}[\varphi(x-y)-\varphi(x)] d x\right) d y \\
& =\int_{N-1<|y|<N+1}+\int_{|y| \leq N-1}=I_{1}+I_{2} .
\end{aligned}
$$

It is easy to check that $I_{2}=0$. For $I_{1}$ we have

$$
\left|I_{1}\right| \leq 2\|\varphi\|_{L^{1}} \int_{N-1<|y|<N+1}|k(y)| d y \leq c\|\varphi\|_{L^{1}}\|\Omega\|_{L^{1}(\Sigma)} N^{-1} .
$$

Therefore,

$$
\int_{\mathbf{R}^{n}} \delta(x) d x=\lim _{N \rightarrow \infty} \int_{|x|<N} \delta(x) d x=0,
$$

as we wanted to show.

3. Proof of the main result. First of all, we shall show in Proposition (3.4) that $K_{\eta}(f)(x)$ and $K(f)(x)$ are defined almost everywhere on $\mathbf{R}^{n}$. For this purpose we shall need the next two lemmas.

(3.1) Lemma. Let $1<p \leq r<\infty$. Then for $(v, u)$ belonging to $A\left(p, p(r / p)^{\prime}\right)$,

$$
\int u(x)^{-p^{\prime}}(1+|x|)^{-n p^{\prime} / r^{\prime}-p^{\prime} / r} d x<\infty .
$$

The proof is simple and will not be given.

(3.2) Lemma. If $(v, u) \in A\left(p, p(r / p)^{\prime}\right), 1 \leq p \leq r<\infty$, then

$$
\|f\|_{L^{1}\left((1+|x|)^{\left.-n / r^{\prime}-1 / r\right)}\right.} \leq c\|f\|_{L^{p}\left(u^{p}\right)},
$$

where the constant $c$ does not depend on $f$.

P r o of. If $1<p \leq r$, by Hölder's inequality we get

$$
\begin{aligned}
& \int|f(x)|(1+|x|)^{-n / r^{\prime}-1 / r} d x \\
& \quad \leq\left(\int|f(x)|^{p} u(x)^{p} d x\right)^{1 / p}\left(\int u(x)^{-p^{\prime}}(1+|x|)^{-n p^{\prime} / r^{\prime}-p^{\prime} / r} d x\right)^{1 / p^{\prime}} .
\end{aligned}
$$


Then, by Lemma (3.1), it follows that (3.3) holds. In the case $p=1$, we observe that $(v, u) \in A\left(1, r^{\prime}\right)$ implies that $(1+|x|)^{-n / r^{\prime}-1 / r} \leq c u(x)$ a.e. Thus (3.3) also holds in this case.

We observe that Lemma (3.2) implies, in particular, that a function belonging to $L^{p}\left(u^{p}\right)$ with $(v, u)$ in $A\left(p,\left(p(r / p)^{\prime}\right)\right.$ belongs locally to $L^{1}$.

(3.4) Proposition. Let $k$ be an $L^{r}$-Dini singular integral kernel. If $f$ belongs to $L^{1}\left((1+|x|)^{-n / r^{\prime}-1 / r}\right)$, then the truncated singular integral $K_{\eta}(f)(x)$ and the singular integral $K(f)(x)$ exist for almost every $x$. In particular, by Lemma (3.2), this holds if $f \in L^{p}\left(u^{p}\right),(v, u) \in A\left(p, p(r / p)^{\prime}\right)$ and $1 \leq p \leq r<\infty$.

Proof. Let $T>1>\eta$. For $|x|<T$, we set

$$
I(x)=\int_{|y|>3 T}|k(x-y)||f(y)| d y .
$$

Then, integrating $I(x)$, we get

$$
\begin{aligned}
\int_{|x|<T} I(x) d x & \leq c \int_{|x|<T}\left(\int_{|y|>3 T}|y|^{-n}|\Omega(x-y)||f(y)| d y\right) d x \\
& =c \int_{|y|>3 T}|f(y)||y|^{-n}\left(\int_{|x|<T}|\Omega(x-y)| d x\right) d y .
\end{aligned}
$$

By Hölder's inequality and enlarging the domain of integration, we obtain

$$
\int_{|x|<T}|\Omega(x-y)| d x \leq c\left(\int_{|y|-T<|z|<|y|+T}|\Omega(z)|^{r} d z\right)^{1 / r} .
$$

Recalling that $\Omega(z)$ is a homogeneous function of degree zero we have

$$
\int_{|x|<T}|\Omega(x-y)| d x \leq c^{\prime}\|\Omega\|_{L^{r}(\Sigma)}|y|^{(n-1) / r} .
$$

Thus, by Lemma (3.2) it follows that

$$
\begin{aligned}
\int_{|x|<T} I(x) d x & \leq c \int_{|y|>3 T}|f(y)||y|^{-n+(n-1) / r} d y \\
& \leq c^{\prime \prime} \int|f(y)|(1+|y|)^{-n / r^{\prime}-1 / r} d y<\infty
\end{aligned}
$$

which shows that $I(x)$ is finite almost everywhere on $|x|<T$. On the other hand, since $f$ belongs locally to $L^{1}$ as we observed after the proof of Lemma (3.2), we find that

$$
\int_{\substack{|x-y|>\eta \\|y|<3 T}} k(x-y) f(y) d y
$$

